\title{
Sobrecarga familiar, apoyo social y salud comunitaria en cuidadores de personas con trastorno mental grave*
}

\section{Family burden, social support and community health in caregivers of people with serious mental disorder \\ Sobrecarga familiar, apoio social e saúde comunitária em cuidadores de pessoas com transtorno mental grave}

José Javier Navarro Pérez ${ }^{1}$ Ángela Carbonell Marqués ${ }^{1}$

Cómo citar este artículo:

Navarro Pérez JJ, Carbonell Marqués A. Family burden, social support and community health in caregivers of people with serious mental disorder. Rev Esc Enferm USP. 2018;52:e03351. DOI: http://dx.doi.org/10.1590/S1980-220X2017029403351

*Extraído de la tesis: "Factores asociados a cuidado familiar de una persona con Trastorno Mental Grave”, Universidad de Valencia, 2016.

${ }^{1}$ Universidad de Valencia, Facultad de Ciencias Sociales, Departamento de Trabajo Social

y Servicios Sociales, Valencia, España.
Autor correspondiente:

Ángela Carbonell Marqués

Facultad de Ciencias Sociales,

Universidad de Valencia

Avenida dels Tarongers, 4b, Despacho 1D19

46022 - Valencia, España

angela.carbonell@uv.es

\begin{abstract}
Objective: To identify caregivers' level of burden and analyze the factors associated with family care in mental health. Method: A cross-sectional descriptive study was conducted between January and July 2016. A sample of caregivers from seven public institutions and an association of relatives and patients was evaluated by identifying their burden and the contributing factors for reduction of these levels through the Zarit Burden Inventory instrument. The study was conducted according to recommendations of the ethics committees of the participating institutions. Results: Participation of 107 caregivers. The main contributions refer that caregivers' active participation in associative dynamics, their attendance at psychoeducational activities and territorial connection to metropolitan areas with community resources decrease their level of burden. Conclusion: Community nursing is highly important and responsible for preventing levels of burden and increasing health levels. In addition, many proposals are formulated in order to favor social support networks by combining treatments and increasing public health programs in contact with the community.
\end{abstract}

\section{DESCRIPTORS}

Mental Disorders; Caregivers; Family; Social Support; Community Health Nursing. 


\section{INTRODUCCIÓN}

E1 trastorno mental grave (TMG) engloba diversos diagnósticos psiquiátricos de duración prolongada y que conllevan un alto grado de discapacidad física, psíquica y social como consecuencia de la enfermedad, reduciendo la calidad de vida de la persona afectada y la de su familia ${ }^{(1)}$. Autores $^{(2)}$ describen este tipo de enfermedad como uno de los predictores de riesgo de dependencia, pues conlleva una gran pérdida de autonomía y requiere de atención y asistencia constante por parte de otra persona para llevar a cabo las actividades básicas e instrumentales de la vida diaria. Es por ello que, este colectivo de personas, en muchas ocasiones, necesita atención y soporte constante por la discapacidad que produce la enfermedad. Esto unido a la falta de recursos, da lugar a que el cuidado de la persona con trastorno mental grave recaiga fundamentalmente en manos de la familia, la cual se posiciona como dispensadora de cuidados y responsable del bienestar de sus miembros ${ }^{(3)}$. Por lo general, dicho cuidado reitera sobre un solo miembro de la familia que, casi en exclusiva, suele ser una mujer.

La función principal del cuidador/a familiar es ayudar en la satisfacción de las necesidades básicas e instrumentales de la vida diaria de la persona en situación de dependencia ${ }^{(4)}$. El/la cuidador/a principal se expone, en el día a día, con una gran variedad de retos y factores de estrés, sufriendo una pérdida de control personal que da lugar a alteraciones físicas y emocionales que pueden desembocar en el llamado "Síndrome del cuidador"(5), enfrentándose a una gran variedad de factores de riesgo que someten su bienestar. Además, esta patología, integra una serie de factores personales y laborales negativos que afectan al bienestar del cuidador/a informal, dando lugar a sensaciones de cansancio emocional, despersonalización e incluso falta de realización personal fuera del cuidado familiar. Esto puede estar motivado por la pérdida del trabajo y de oportunidades laborales que puede sufrir una persona que dedica la mayor parte de su tiempo al cuidado de un familiar, lo que soporta un empeoramiento de la situación económica debido a los costes directos y al descenso de la productividad familiar ${ }^{(6)}$. Esta gran inversión del tiempo de la persona cuidadora, tiene como consecuencia la reducción del ocio y las relaciones sociales, y proyecta a su alrededor altas fragilidades y la sitúa vulnerable en torno a situaciones de aislamiento y exclusión social ${ }^{(7)}$.

La atención de una persona diagnosticada de algún trastorno mental grave supone un desgaste económico, psíquico y personal importante para aquellas personas responsables del cuidado diario de la misma ${ }^{(8)}$. La literatura científica destaca que el cuidado de personas con trastornos mentales graves supone una carga más pesada que el cuidado de personas que tienen otro tipo de enfermedad ${ }^{(9)}$. Algunos de los factores que inciden en este deterioro son la sobrecarga del cuidado de una persona enferma, la falta de formación específica para atenderla de manera adecuada, así como la preparación para afrontar las situaciones que el mismo cuidado conlleva ${ }^{(10)}$. Todo esto, unido al estigma y rechazo social de la propia enfermedad, así como la asociación que se hace de esta con la violencia. Se atiende a la falsa creencia social de que la persona que padece TMG es agresiva por la naturaleza de la enfermedad, y por tanto, considerarse esto como factor de riesgo para el cuidado. Esta cuestión viene potenciada por el modo en que afecta a las relaciones interpersonales, al desequilibrio entre el coste de la enfermedad y la creencia de que las conductas violentas surgen como una forma de mostrar la desilusión, el estigma y el rechazo hacia el propio trastorno ${ }^{(11)}$.

Diferentes autores han tratado de evaluar el impacto de las variables sociodemográficas y clínicas, tanto de las personas con trastorno mental grave como sus cuidadores/as, y la sobrecarga. Los resultados de estas investigaciones asocian un mayor nivel de carga en función de variables como el sexo, el parentesco, la tenencia de un trabajo remunerado, el tipo de enfermedad mental, etc. Por otro lado, estos autores correlacionan positivamente la edad del/la cuidador/a, los años de evolución de la enfermedad, el número de ingresos hospitalarios y el grado de discapacidad y dependencia como variables asociadas a la sobrecarga del cuidador/a princi$\mathrm{pal}^{(12-13)}$. Asimismo, el apoyo social se considera como un factor de protección para hacer frente a las situaciones del cuidado, ya que incide positivamente en el bienestar psicológico y la calidad de vida del mismo. Según los estudios, los/as cuidadores/as experimentan mayor carga cuando el apoyo social percibido es menor ${ }^{(14-16)}$.

Los modelos de atención a las personas en situación de dependencia han ido transformándose con el paso del tiempo, tratando de adaptarse a las necesidades, para dar respuesta a los problemas psíquicos y psicosociales de este colectivo. En España, el modelo social de atención a las personas en situación de dependencia, ha favorecido el mantenimiento de la persona enferma en el contexto familiar y social, provocando que las familias se constituyan como el eje principal de soporte y cuidado. La enfermería se ha posicionado como área responsable en proporcionar educación sanitaria y potenciar la prestación de cuidados en las mejores condiciones posibles, no sólo a las personas enfermas y también a sus cuidadores/as, valorando las repercusiones que esta labor pueda tener sobre él/ella ${ }^{(17)}$. En este sentido, la enfermería comunitaria es clave en el proceso de rehabilitación psicofísica y de apoyo e integración social de las personas enfermas, como también de sus cuidadores ${ }^{(18)}$.

De aquí surge la necesidad de investigar la situación de las personas que dedican su vida al cuidado de $\operatorname{otra}^{(19-20)}$. Por ello, el objetivo de la presente investigación seria doble; por una lado, identificar el nivel de sobrecarga de los y las cuidadores/as y por otro, analizar los factores asociados con el cuidado familiar en salud mental.

\section{MÉTODO}

\section{TIPO DE ESTUDIO}

Se realizó un estudio descriptivo transversal y se utilizó la encuesta como técnica de recolección de datos. El trabajo se realizó entre los meses de enero y julio del año 2016 en siete dispositivos sanitarios de atención a la salud mental de la provincia de Valencia y una asociación de familiares y pacientes. 


\section{PARTICIPANTES}

La muestra en la que se realizó el estudio estuvo compuesta por 107 cuidadores/as que cumplían los siguientes criterios de inclusión: Cuidadores/as de personas con TMG incluidas en el sistema sanitario público de atención a la Salud Mental de la Comunidad Valenciana; Residir en la provincia de Valencia; Mantener lazos de parentesco con la persona enferma; Tener más de 18 años; Ejercer el rol de cuidador/a principal, adoptando funciones de asistencia y supervisión en las actividades básicas y/o instrumentales de la vida diaria de la persona con problemas de Salud Mental; Ausencia de remuneración por la labor que realizan; Ejercer el cuidado por un tiempo superior a seis meses.

Asimismo, fueron criterios de exclusión desestimar la participación en el estudio, ser cuidadores/as de personas con diagnósticos no clasificados como enfermedad mental grave (discapacidad intelectual, alteraciones conductuales o del desarrollo psicomotor) o por no estar cumplimentados correctamente.

\section{INSTRUMENTOS}

Debido a la extensa literatura científica que ha hecho uso y ha estudiado la validez de esta escala ${ }^{(21)}$, se escogió el Zarit Burden Inventory/Test de Zarit ${ }^{(22-23)}$ como instrumento de recogida de datos. Esta herramienta tiene por objeto evaluar el grado de sobrecarga que padecen los/as cuidadores/as de personas con demencia, no obstante, ha sido utilizado también para cuidadores/as de personas con enfermedad mental por la similitud en la cronicidad de ambos colectivos $^{(24-25)}$. La escala obtuvo, para el presente estudio, un coeficiente de Alfa de Cronbach de 0,92.

Para analizar las características sociodemográficas de las personas cuidadoras e identificar la relación de estas y el nivel de sobrecarga, se recogieron las variables sociodemográficas y clínicas con más presencia en la literatura científica y que, según los/as autores/as se relacionan con el Síndrome del cuidador: 1) Variables que describen al cuidador/a: edad, sexo, estado civil, parentesco que tiene con la persona enferma, tenencia de trabajo remunerado, tenencia de enfermedad crónica, asociacionismo activo, asistencia a charlas y talleres psicoeducativos familiares, entorno geográfico y tipo de atención recibida; y 2) Variables que describen a la persona con TMG: edad, sexo, enfermedad diagnosticada, años de evolución de la enfermedad, grado de discapacidad reconocido y dispositivo de atención a la Salud Mental al que asisten.

\section{Procedimiento}

Teniendo en cuenta que el sistema de atención a la Salud Mental español no atiende de manera directa a los/as cuidadores/as, la recogida de información se llevó a cabo en siete dispositivos incluidos en el sistema sanitario público de atención a la Salud Mental de la Comunidad Valenciana, en la provincia de Valencia, y una asociación de familiares y pacientes. Las enfermeras comunitarias aprovecharon las entrevistas a las que asistían los/as usuarios/as con algún familiar y debían identificar, desde su juicio profesional, aquellos que ejercían el cuidado principal de la persona atendida.
Se concertó una entrevista individualizada con cada participante del estudio. Las evaluaciones fueron realizadas por enfermeras comunitarias entrenadas para aplicar los instrumentos. A su vez, en aquellos casos en los que se detectó un nivel de sobrecarga elevado, se informó de la existencia de programas psicoeducativos para familiares.

El desarrollo de esta investigación se llevó a cabo de acuerdo a las recomendaciones de los comités de ética de las instituciones participantes y cumplió con los estándares nacionales (Ley 14/2007, de 3 de julio, de Investigación biomédica) e internacionales de ética en investigación. Los resultados de la presente investigación son extraídos de una investigación más amplia aprobada por la Comisión de Ética e Investigaciones Experimentales de la Facultad de Ciencias Sociales de la Universidad de Valencia, con la referencia 000217/UV-Soc/2016. Las personas que cumplieron los criterios de inclusión para este estudio recibieron previamente la necesaria información y prestaron consentimiento de participación voluntaria por escrito, de acuerdo con la Declaración de Helsinki (2013). Asimismo, se explicó a los y las participantes la posibilidad de interrumpir o rechazar su participación en cualquier momento.

\section{ANÁLISIS DE DATOS}

Para el análisis cuantitativo, en primer lugar, se realizó un estudio descriptivo de los datos para identificar las características del/la cuidador/a y de la persona cuidada a partir de variables sociodemográficas y clínicas. Asimismo, se identificó la relación entre las variables que definen al/la cuidador/a y la persona cuidada y la sobrecarga. Se utilizaron tablas de contingencia y pruebas Chi-Cuadrado para comprobar si existían diferencias estadísticamente significativas entre la sobrecarga y las variables nominales, y un análisis de correlaciones para demostrar la existencia de una relación lineal entre el nivel de sobrecarga y las variables cuantitativas. Finalmente, se efectuó una regresión lineal con las variables que tuvieron una relación significativa con el nivel de sobrecarga en los análisis anteriores, con la finalidad de definir un modelo de predicción de la sobrecarga. Al tratarse de variables nominales, se recodificaron como variables Dummy (ficticias) para poder incorporarlas al modelo de regresión. El proceso de análisis de datos se realizó mediante el programa informático IBM SPSS Statistics 25. Para todas las pruebas realizadas se consideró $\mathrm{p}<, 05$ como el nivel de significación estadística.

\section{RESULTADOS}

\section{CARACTERÍ́STICAS DEL/LA CUIDAdor/A}

La muestra total del estudio estuvo compuesta por un total de 107 cuidadores/as familiares de personas con Trastorno Mental Grave atendidos en siete dispositivos públicos de atención a la Salud Mental y una asociación de familiares y pacientes (Tabla 1). El 71\% eran mujeres y el 29\% eran hombres, con edades comprendidas entre 16 y 89 años ( $M=60,67$; $\mathrm{SD}=13,83)$. Según el estado civil, el 51,4\% estaban casados/ as, el 15\% divorciados/as, el 21,5\% eran viudos/as y el 12,1\% solteros/as. E1 41,1\% de las personas encuestadas tenían un 
trabajo remunerado y el mismo porcentaje afirmó tener una enfermedad crónica. La mayor parte de los/as cuidadores/ as cumplían el rol de padre/madre de la persona con TMG $(78,5 \%)$, frente al $12,1 \%$ de hermanos/as, $4,5 \%$ de hijos/as, $3,7 \%$ de esposos/as y el 0,9\% de tío/a. El 48,6\% de los/as cuidadores/as que participaron en el estudio habían asumido anteriormente el cuidado de otra persona. Este mismo porcentaje habían asistido a charlas psicoeducativas para familiares en los dispositivos de Salud Mental y un 15\% participaban en actividades asociativas para familiares y personas con TMG.

Los sujetos diagnosticados con patologías mentales crónicas, en su mayoría, eran de sexo masculino $(72,4 \%)$ y tenían una edad promedio de 38,64 años (SD=12,63) que osciló entre los 16 y los 80 años. Todas estaban diagnosticadas de algún Trastorno Mental Grave, donde destacaron la esquizofrenia $(63,6 \%)$, el trastorno bipolar $(15,9 \%)$, trastornos de personalidad (10,3\%), distimia y depresión crónica (4,7\%), y con menores porcentajes TOC y esquizofrenia, patología dual y trastorno esquizoafectivo, con un 1,9\% cada uno. E1 50,5\% de estas personas contaban con un Reconocimiento del Grado de Discapacidad con un $65 \%$ o superior, mientras que el 19,6\% no la tenían reconocida o no la habían solicitado. La media de años de evolución de la enfermedad fue de 17,28 (SD=13,18) y osciló entre los tres meses y los 56 años de duración.

Tabla 1 - Características sociodemográficas del/la cuidador/a y de la persona cuidada -Valencia, España, 2016.

\begin{tabular}{|c|c|c|c|}
\hline \multicolumn{2}{|c|}{ Características del/la cuidador/a } & \multicolumn{2}{|c|}{ Características de la persona cuidada } \\
\hline Edad (años) & $60,67 \pm 13,83$ & Edad (años) & $38,67 \pm 12,63$ \\
\hline Sexo (mujer) & $71 \%$ & Sexo (hombre) & $72 \%$ \\
\hline \multicolumn{4}{|l|}{ Estado civil } \\
\hline Casado/a & $51,4 \%$ & Diagnóstico & \\
\hline Divorciado/a & $15,0 \%$ & Esquizofrenia & $63,6 \%$ \\
\hline Viudo/a & $21,5 \%$ & Trastorno Bipolar & $15,9 \%$ \\
\hline Soltero/a & $12,1 \%$ & Depresión & $4,7 \%$ \\
\hline Trabajo remunerado (sí) & $41,1 \%$ & TOC y Esquizofrenia & $1,9 \%$ \\
\hline Enfermedad crónica (sí) & $41,1 \%$ & T. Personalidad & $10,3 \%$ \\
\hline Parentesco con la persona & & Patología dual & $1,9 \%$ \\
\hline Esposo/a & $3,7 \%$ & T. Esquizoafectivo & $1,9 \%$ \\
\hline Hermano/a & $12,1 \%$ & & \\
\hline Padre/Madre & $78,5 \%$ & R. Grado Discapacidad & \\
\hline $\mathrm{Hijo/a}$ & $4,7 \%$ & No reconocido & $19,6 \%$ \\
\hline Tío/a & $0,9 \%$ & Menos del $65 \%$ & $29,9 \%$ \\
\hline Asociacionismo activo (sí) & $15 \%$ & $65 \%$ o más & $50,5 \%$ \\
\hline Cuidado previo de otro familiar (sí) & $48,6 \%$ & Años evolución de la enfermedad & $17,28 \pm 13,18$ \\
\hline Psicoeducativos (sí) & $48,6 \%$ & & \\
\hline
\end{tabular}

\section{SobreCARGA DEL/A CUIDADOR/A}

Al aplicar la escala de Zarit, el 73,8\% de las personas encuestadas obtuvieron un nivel de sobrecarga intensa, el 9,3\% sobrecarga leve y el 16,4\% no mostraron niveles de sobrecarga.

\section{RELACIÓN ENTRE EL NIVEL DE SOBRECARGA Y LAS VARIABLES SOCIODEMOGRÁFICAS Y CLÍNICAS}

En las pruebas Chi-cuadrado realizadas se hallaron factores que no obtuvieron una relación significativa de $\mathrm{p}<, 05$ con el nivel de sobrecarga, tales como las variables sociodemográficas del/la cuidador/a: sexo, edad, parentesco, estado civil, cuidado previo; así como el sexo, edad, diagnóstico, reconocimiento del Grado de Discapacidad y años de evolución de la persona cuidada. Se mostraron relaciones significativas entre el grado de sobrecarga y las variables tipo de intervención familiar $(p=, 000)$, asociacionismo $(p=, 000)$, psicoeducativos $(p=, 000)$ y entorno geográfico $(\mathrm{p}=, 021)$. De este modo, aquellos/as cuidadores/as que recibían una atención continuada en un recurso de atención a la salud mental, que participaban activamente en alguna asociación, que habían asistido a charlas psicoeducativas para familiares o que vivían en áreas metropolitanas, presentaron un grado de sobrecarga inferior al resto de los/as participantes. En el análisis de correlaciones de Pearson no se halló ninguna relación entre las variables cuantitativas y el grado de sobrecarga del/la cuidador/a.

\section{Modelo de Regresión LineAl}

Al utilizar las variables que resultaron tener una relación significativa con el grado de sobrecarga del/la cuidador/a (tipo de atención, asociacionismo, psicoeducativos y entorno geográfico), el análisis de regresión lineal creó dos modelos predictores de la sobrecarga (Tablas 2-4):

El primer modelo obtuvo una significación $p=, 000, y$ explicaba la no asistencia a charlas psicoeducativas como predictores de la sobrecarga. De este modo, aquellas personas que acudieron a estas actividades tuvieron 19,4 puntos menos de sobrecarga que los que nunca asistieron.

En el segundo modelo, el asociacionismo activo y la asistencia a charlas y actividades psicoeducativas resultaron ser factores predictores de una menor sobrecarga, encontrándose que aquellos/as cuidadores/as que habían asistido a estos programas $(\mathrm{p}=, 00)$ tuvieron 15 puntos menos de sobrecarga que los que nunca asistieron. Asimismo, las personas cuidadoras que participaron activamente en una asociación $(\mathrm{p}=, 001)$ tuvieron 14,4 puntos menos de sobrecarga que las que nunca participaron. 
Tabla 2 - Resumen del modelo de regresión de la sobrecarga del/ la cuidador/a en una muestra de cuidadores familiares - Valencia, España, 2016.

\begin{tabular}{lcccc}
\hline Modelo & $\mathbf{R}$ & R cuadrado & $\begin{array}{c}\text { R cuadrado } \\
\text { ajustado }\end{array}$ & $\begin{array}{c}\text { Error típ. de la } \\
\text { estimación }\end{array}$ \\
\hline $\mathbf{1}$ &, $558^{\mathrm{a}}$ &, 312 &, 305 & 14,562 \\
$\mathbf{2}$ &, $619^{\mathrm{b}}$ &, 383 &, 371 & 13,853 \\
\hline
\end{tabular}

${ }^{a}$ Predictores: (Constante), Psicoeducativos

b Predictores: (Constante), Psicoeducativos, Asociacionismo

Tabla 3 - Resumen del ANOVA de la sobrecarga del/la cuidador/a en una muestra de cuidadores familiares - Valencia, España, 2016.

\begin{tabular}{llccccc}
\hline \multicolumn{6}{c}{ ANOVA $^{\text {a }}$} \\
\hline Modelo & $\begin{array}{c}\text { Suma de } \\
\text { cuadrados }\end{array}$ & Gl & $\begin{array}{c}\text { Media } \\
\text { cuadrática }\end{array}$ & F & Sig. \\
\hline $\mathbf{1}$ & Regresión & 10076,766 & 1 & 10076,766 & 47,520 &, $000^{\mathrm{b}}$ \\
& Residuo & 22265,440 & 105 & 212,052 & & \\
& Total & 32342,206 & 106 & & & \\
$\mathbf{2}$ & Regresión & 12383,435 & 2 & 6191,717 & 32,263 &, $000^{\mathrm{c}}$ \\
& Residuo & 19958,771 & 104 & 191,911 & & \\
& Total & 32342,206 & 106 & & & \\
\hline
\end{tabular}

a Variable dependiente: Sobrecarga del/la cuidador

${ }^{b}$ Predictores: (Constante), Psicoeducativos

c Predictores: (Constante), Psicoeducativos, Asociacionismo

Tabla4-Coeficientes de regresión de la sobrecarga del/la cuidador/a en una muestra de cuidadores familiares - Valencia, España, 2016.

\begin{tabular}{llccccc}
\hline \multirow{2}{*}{ Modelo } & & $\begin{array}{c}\text { Coeficientes no } \\
\text { estandarizados }\end{array}$ & $\begin{array}{c}\text { Coeficientes } \\
\text { estandarizados }\end{array}$ & t & Sig. \\
\cline { 3 - 8 } & B & Error típ. & Beta & B & $\begin{array}{c}\text { Error } \\
\text { típ. }\end{array}$ \\
\hline $\mathbf{1}$ & (Constante) & 75,782 & 1,964 & & 38,594 &, 000 \\
& Psicoeducativos & $-19,416$ & 2,817 &,- 558 & $-6,893$ &, 000 \\
$\mathbf{2}$ & (Constante) & 75,782 & 1,868 & & 40,569 &, 000 \\
& Psicoeducativos & $-14,976$ & 2,970 &,- 431 & $-5,043$ &, 000 \\
& Asociacionismo & $-14,431$ & 4,162 &,- 296 & $-3,467$ &, 001 \\
\hline
\end{tabular}

${ }^{a}$ Variable dependiente: Sobrecarga del/la cuidador

Atendiendo el segundo modelo obtenido en la regresión lineal, la sobrecarga del/la cuidador/a de una persona con Trastorno Mental Grave quedaría representado de la siguiente manera:

$$
\mathrm{Y}^{1}=\mathrm{B}_{0}+\mathrm{B}_{1} \mathrm{X} 1+\mathrm{B}_{2} \mathrm{X}_{2}
$$

SOBRECARGA $=75,78+(-14,976)$ Psicoeducativo + $(-14,431)$ Asociacionismo

\section{DISCUSIÓN}

La afectación a las esferas biológica, psicológica y social de la persona a causa del trastorno mental grave posiciona a sujetos que tienen este tipo de enfermedad como población susceptible para la recepción de cuidado y asistencia a largo plazo $^{(1-2)}$. Los estudios existentes hacen referencia a que las personas que cuidan a otra con trastorno mental grave, presentan altos niveles de carga, lo cual deriva en una serie de factores psicológicos, físicos, económicos y sociales que repercuten de manera negativa en la persona cuidadora ${ }^{(8)}$. La presente investigación ha identificado variables asociadas con la percepción de sobrecarga de los/as cuidadores/as familiares de personas con trastorno mental grave.

El perfil predominante es el de una mujer, con una media de edad de 60 años, casada, habitualmente madre de la persona con enfermedad mental y que no cuenta con un trabajo remunerado. El presente estudio evidencia que la elevada feminización es una de las características principales que definen el cuidado formal e informal, el $71 \%$ de las personas que ejercían el cuidado eran mujeres, coincidiendo con autores ${ }^{(2,19)}$ se hace evidente y necesario el enfoque desde la perspectiva de género en el cuidado informal.

La literatura científica defiende la relación entre las variables sociodemográficas del cuidador como de la persona cuidada y las características propias de la enfermedad, con el nivel de carga y los factores de riesgo, siendo las variables más destacadas en los estudios: la edad, el sexo, la ocupación y el nivel de estudios de la persona cuidadora, así como el parentesco, la edad, los años de evolución de la enfermedad y la severidad de los síntomas de la persona cuidada ${ }^{(12-13)}$. Sin embargo, debido a los elevados niveles de carga hallados en la mayor parte de los sujetos a estudio, las pruebas estadísticas realizadas para el análisis de los datos cuantitativos señalan que la sobrecarga, en esta muestra, no guarda una relación significativa con ninguna de estas variables, sino que depende del apoyo social - formal e informal - que recibe la persona cuidadora.

Diferentes investigaciones ${ }^{(14-16)}$ destacan la importancia del apoyo social como variable que modera el impacto negativo del desempeño del rol del cuidador, como un amortiguador del estrés. Para el presente estudio, el acceso a una atención continua por parte de profesionales, el asociacionismo activo y la asistencia a charlas y talleres psicoeducativos para familiares determinó los niveles de sobrecarga de los y las cuidadores/as. Asimismo, estas dos últimas variables fueron capaces de definir un modelo de predicción de la sobrecarga donde, coincidiendo con estos autores, las personas que contaban con mayor apoyo social mostraron menores niveles de sobrecarga por el cuidado que realizan.

Investigaciones recientes han destacado el elevado impacto de la familia como canal de acceso a la socialización ${ }^{(26)}$. Esta dimensión favorece el afrontamiento de los miembros del núcleo familiar ${ }^{(27)}$, destacando entre estos el apoyo social proporcionado por los padres y las potencialidades generadas a partir de la red de relaciones. Nuestros resultados abordan esta cuestión, ya que las posibilidades de apoyo social se construyeron a partir de la inmersión tanto de familiares como de enfermos en actividades comunitarias. Coincidimos con otros análisis $^{(28)}$ que constatan la importancia de una afectividad estable en las relaciones familiares cotidianas, como factor clave para el ajuste social y la reducción de carga emocional.

Estudios $^{(29)}$ nos informan de que la falta de apoyo social ante situaciones estresantes, repercute en la estabilidad de la persona que necesita ayuda y por consiguiente en la de los que le rodean o con quienes conviven y relacionaron el compromiso de los padres en la asistencia continuada de actividades psicoeducativas con bajo estrés soportado. En esta línea, nuestros resultados muestran similitudes ya que los familiares que asistieron a actividades de apoyo para la enfermedad, hallaron menor sobrecarga. 
Paralelamente, las áreas metropolitanas integran mayores y mejores posibilidades de integración tanto de las personas con TMG como de sus familiares, ya que la oferta es más amplia y también diversificada. En esta línea, nuestros resultados se aproximaron a los ofrecidos por otros autores, pues las áreas rurales o aquellas con menor oferta, el nivel de sobrecarga e incluso de mortalidad, fue mayor que en las grandes ciudades o con mayores posibilidades de ocio prosocial, diversificación en la oferta o áreas recreativas. La comunidad tiene alta ascendencia en para el desarrollo y bienestar colectivo, por tanto, en la creación de redes de apoyo social mejora la calidad de vida y salud mental de sus miembros ${ }^{(30)}$.

La enfermería se enfrenta ante el desafío y la oportunidad de hacer que el cuidado sea prioritario y significativo para la salud mental, asegurando una continuidad de atención y apoyo formal capaz de responder de manera sensible y eficaz a las necesidades de sujetos y de sus cuidadores/as ${ }^{(17-18)}$.

\section{CONCLUSIÓN}

Los trastornos mentales graves se consideran un problema de salud pública a nivel mundial, que repercute principalmente en la calidad de vida de las personas que lo sufren y sus familias. Las personas con trastorno mental grave requieren, en ocasiones, de apoyo y soporte para la realización de las tareas cotidianas, y la persona que asume el rol del cuidado cuenta con una serie de factores de riesgo asociados a la carga que derivan de esta labor. Por ello, los/ as cuidadores/as informales son un colectivo en riesgo, al encontrarse en situaciones de vulnerabilidad, aislamiento y en ciertos casos, en exclusión.

En el presente estudio se hallaron limitaciones en relación al procedimiento de selección de los y las participantes y de la muestra final. Se recurrió a profesionales de la Enfermería comunitaria por ser ellos/as quienes se encuentran en contacto habitual con los pacientes y sus cuidadores con objeto de su estudio empírico. La selección de la muestra se realizó en base a tres filtros: acompañantes de las personas atendidas por los dispositivos que cumplieran una serie de criterios de inclusión descritos anteriormente, que ejercieran, a juicio profesional, la función de cuidador/a y que aceptaran participar voluntariamente en el estudio. De dicha selección surgieron dos problemas: 1) la muestra de cuidadores/as se seleccionó de manera subjetiva y a razón del/la profesional que suministró los cuestionarios, y 2) únicamente se proporcionaron cuestionarios a aquellos/as que acompañaron a la persona diagnosticada al recurso, lo que impidió el acceso a una mayor muestra.

Sin embargo, los hallazgos de este estudio destacan los beneficios de la creación espacios asociativos de interacción comunitaria y apoyo mutuo, y la importancia de los talleres psicoeducativos para comprender los procesos de la enfermedad, desarrollar estrategias de afrontamiento y empoderar tanto a los/as cuidadores/as como a las personas cuidadas.

Por tanto, es necesario que las autoridades de salud pública centren sus esfuerzos en el bienestar social de la población a la que atienden. En este sentido, sería recomendable atender a las siguientes propuestas: Favorecer redes de apoyo coordinadas entre las áreas de educación para la salud, enfermería y servicios a la comunidad e incrementar los programas de salud mental en la comunidad, favoreciendo conjuntamente tratamientos combinados para la recuperación y estabilidad de personas con TMG y a su vez, liberar de la carga a las personas cuidadoras. Asimismo, el presente estudio suscita la necesidad de promover hospitales de día de base comunitaria a los que los usuarios puedan acudir tanto a recibir tratamiento de salud como a ampliar su circuito social; colaborar desde la administración sanitaria, en la creación tanto de asociaciones de familiares de personas con TMG como de usuarios o mixtas, con objeto de dinamizar las redes de apoyo disponible y generar nuevas, así como implementar programas complementarios a los articulados desde salud pública; y conectar zonas rurales o escasos recursos con áreas geográficas metropolitanas para la reducción de los niveles de sobrecarga familiar tanto de cuidadores como en la recuperación y estabilización psiquiátrica de los enfermos.

En definitiva, el asociacionismo, los programas de apoyo social en la comunidad y las actividades sociosanitarias rehabilitadoras, se establecen como un factor protector, que en ausencia de estructura para su desarrollo pueden proyectar prácticas de riesgo tanto para cuidadores como para los enfermos, y que afecten al deterioro comunitario.

\section{RESUMEN}

Objetivo: Identificar el nivel de sobrecarga de los cuidadores y analizar los factores asociados con el cuidado familiar en salud mental. Método: Se realizó un estudio descriptivo transversal realizado entre los meses de enero y julio del año 2016, en el que se evalúa una muestra de cuidadores de siete dispositivos públicos y una asociación de familiares y pacientes identificando la sobrecarga de los mismos y los factores que contribuyen a reducir estos niveles, a través del instrumento Zarit Burden Inventory. El estudio se llevó a cabo de acuerdo a las recomendaciones de los comités de ética de las instituciones participantes. Resultados: Participaron 107 cuidadores. Las principales aportaciones refieren que la participación activa del cuidador en dinámicas asociativas, la asistencia a actividades psicoeducativas y la vinculación territorial a áreas metropolitanas con recursos comunitarios, disminuyen el nivel de sobrecarga del cuidador. Conclusión: Finalmente, destaca la importancia y responsabilidad de la enfermería comunitaria a efectos de prevenir los niveles de sobrecarga e incrementar los de salud; además, se realizan una serie de propuestas en la línea de favorecer redes de apoyo social, combinar tratamientos e incrementar los programas de salud pública en contacto con la comunidad.

\section{DESCRIPTORES}

Trastornos Mentales; Cuidadores; Familia, Apoyo Social; Enfermería en Salud Comunitaria. 


\section{RESUMO}

Objetivo: Identificar o nível de sobrecarga dos cuidadores e analisar os fatores associados ao cuidado familiar em saúde mental. Método: Foi realizado um estudo descritivo transversal entre os meses de janeiro e julho do ano 2016, no qual foi avaliada uma amostra de cuidadores de sete dispositivos públicos e uma associação de familiares e pacientes identificando a sobrecarga dos mesmos e os fatores que contribuem para reduzir esses níveis, por meio do instrumento Zarit Burden Inventory. O estudo foi realizado de acordo com as recomendações dos comitês de ética das instituições participantes. Resultados: Participaram 107 cuidadores. As principais contribuições relatam que a participação ativa do cuidador em dinâmicas associativas, o comparecimento a atividades psicoeducacionais e o vínculo territorial a áreas metropolitanas com recursos comunitários diminuem o nível de sobrecarga do cuidador. Conclusão: Finalmente, destaca a importância e responsabilidade da enfermagem comunitária com o objetivo de prevenir os níveis de sobrecarga e aumentar os de saúde; além disso, é realizada uma série de propostas no sentido de favorecer as redes de apoio social, combinar tratamentos e aumentar os programas de saúde pública em contato com a comunidade.

\section{DESCRITORES}

Transtornos Mentais; Cuidadores; Família; Apoio Social; Enfermagem em Saúde Comunitária.

\section{REFERENCIAS}

1. Mata B, Delgado P, Resa J, Rodríguez T. Revisión bibliográfica de los cuidados del paciente anciano con trastorno mental grave desde una perspectiva multidisciplinar. Eur J Develop Educa Psychop. 2016;4(1):31-47.

2. De León N, Bagnato MJ, Luzardo M. Proceso de cronificación en el campo de salud mental: índice de cronicidad, concepto y medición. Rev Urug Enferm [Internet]. 2016 [citado 2017 jul. 22];11(1). Disponible en: http://rue.fenf.edu.uy/index.php/rue/article/view/192

3. Bellato R, Araújo L, Dolina J, Musquim C, Corrêa G. The family experience of care in chronic situation. Rev Esc Enferm USP. 2016;50(n. spe):78-85. DOI: http://dx.doi.org/10.1590/S0080-623420160000300012

4. Silva J, Gonzales J, Mas T, Marques S, Partezani R. Sobrecarga y calidad de vida del cuidador principal del adulto mayor. Av Enferm. 2016;34(3):251-8.

5. Echevarría RG, Crespo LG, Crespo BG, Méndez LP, Fernández MA, Martínez D. La atención al cuidador, problema de salud necesario de abordar en los estudios médicos. Edumecentro. 2014;6(3):128-42.

6. Parekh N, Shah S, McMaster K, Speziale A, Yun L, Nguyen D, et al. Effects of caregiver burden on quality of life and coping strategies utilized by caregivers of adult patients with inflammatory bowel disease. Ann Gastroenterol. 2017;30(1):89-95.

7. Ribé JM, Salamero M, Pérez-Testor C, Mercadal J, Aguilera C, Cleris M. Quality of life in family caregivers of schizophrenia patients in Spain: caregiver characteristics, caregiving burden, family functioning, and social and professional support. Int J Psychiatry Clin Pract. 2018;22(1):25-33.

8. Ong HC, Ibrahim N, Wahab S. Psychological distress, perceived stigma, and coping among caregivers of patients with schizophrenia. Psychol Res Behav Manag. 2016;9:211-8.

9. Thunyadee C, Sitthimongkol Y, Sangon S, Chai-Aroon T, Hegadoren KM. Predictors of depressive symptoms and physical health in caregivers of individuals with schizophrenia. Nurs Health Sci. 2015;17(4):412-9.

10. Guerra MD, Zambrano E. Relación entre los problemas de salud de los mayores dependientes y la formación de los cuidadores informales. Enferm Global. 2013;12(32):211-21.

11. Fasihi T, Taghinasab M, Nayeri, TD. The correlation of social support with mental health: a meta-analysis. Elect Pshysic. 2017; 9(9):5212-22.

12. Zhou Y, Rosenheck R, Mohamed S, Ou Y, Ning Y, He H. Comparison of burden among family members of patients diagnosed with schizophrenia and bipolar disorder in a large acute psychiatric hospital in China. BMC Psychiatry. 2016;16(1):283.

13. Geriani D, Savithry KSB, Shivakumar S, Kanchan T. Burden of care on caregivers of schizophrenia patients: a correlation to personality and coping. J Clin Diagn Res. 2015;9(3): VC014.

14. Barrera-Ortiz L, Campos M, Gallardo-Solarte K, Coral-Ibarra RC, Hernández-Bustos A. Social support perceived in people with chronic disease and their family caregivers in five macro regions of Colombia. Rev Univ Salud. 2016;18(1):102-12.

15. Kate N, Grover S, Kulhara P, Nehra R. Relationship of caregiver burden with coping strategies, social support, psychological morbidity, and quality of life in the caregivers of schizophrenia. Asian J Psychiatr. 2013;6(5):380-8.

16. Mao ZH, Zhao XD. The effects of social connections on self-rated physical and mental health among internal migrant and local adolescents in Shanghai, China. BMC Public Health. 2012; 12:97. DOI: 10.1186/1471-2458-12-97

17. De Jacq K, Norful AA, Larson E. The variability of nursing attitudes toward mental illness: an integrative review. Arch Psychiatr Nurs. 2016;30(6):788-96.

18. Wright N, Stickley T. Concepts of social inclusion, exclusion and mental health: a review of the international literature. J Psychiatr Ment Health Nurs. 2013;20(1):71-81.

19. Masana L. Cuidados informales de larga duración en España: retos, miradas y soluciones. Salud Colectiva. 2017;13(2):337-52.

20. Espinoza K, Jofre V. Burden, social support and self-care in informal caregivers. Cienc Enferm. 2012;18(2):23-30.

21. Kahn PV, Wishart HA, Randolph JS, Santulli RB. Caregiver stigma and burden in memory disorders: an evaluation of the effects of caregiver type and gender. Curr Gerontol Geriatr Res. 2016;2016:8316045.

22. Zarit SH, Zarit JM. The memory and behavior problem checklist and the burden interview Pennsilvania; Pennsilvania State University; 1983.

23. Martín M, Salvadó I, Nadal S, Miji LC, Rico JM, Lanz P, et al. Adaptación para nuestro medio de la Escala de Sobrecarga del Cuidador (Caregiver Burden Interview) de Zarit. Rev Gerontol. 1996;6(1):338-46. 
24. Rofail D, Regnault A, le Scouiller S, Lambert J, Zarit SH. Assessing the impact on caregivers of patients with schizophrenia: psychometric validation of the Schizophrenia Caregiver Questionnaire (SCQ). BMC Psychiatry. 2016;16:245. DOI: 10.1186/s12888-016-0951-1

25. Vella SL, Pai N. The measurement of burden of care in serious mental illness: a qualitative review. Aust N Z J Psychiatry. 2012;47(3):222-34.

26. Guo M, Li S, Liu J, Sun F. Family Relations, Social Connections, and Mental Health Among Latino and Asian Older Adults. Res Aging. 2015;37(2):123-47.

27. Paul HA. Helping young mothers and fathers become effective co-parents. Child Fam Behav Ther. 2015;37:243-9.

28. Whitley DM, Kelley SJ, Lamis DA. Depression, social support, and mental health. a longitudinal mediation analysis in African American custodial grandmothers. Int J Aging Hum Dev. 2016;82(2-3):166-87.

29. Lambert EG, Minor KI, Wells JL, Hogan NL. Leave your job at work: the possible antecedents of work-family conflict among correctional staff. Prison J. 2015;95(1):114-34.

30. Silveira LHDC, Rocha, CMF, Zanardo GLDP. O outro lado da porta giratória: apoio comunitário e saúde mental. Psicol Estud. 2016;21(2):325-35. 